\title{
Pratiques
}

Linguistique, littérature, didactique

185-186 | 2020

Lire des documents composites en classe

\section{Linguistiques textuelles et discursives}

Introduction: textual and discursive linguistics

\section{Guy Achard-Bayle}

\section{(2) OpenEdition}

Journals

Édition électronique

URL : http://journals.openedition.org/pratiques/8521

DOI : 10.4000/pratiques.8521

ISSN : 2425-2042

\section{Éditeur}

Centre de recherche sur les médiations (CREM)

\section{Référence électronique}

Guy Achard-Bayle, "Linguistiques textuelles et discursives », Pratiques [En ligne], 185-186 | 2020, mis en ligne le 30 juin 2020, consulté le 15 octobre 2020. URL : http://journals.openedition.org/pratiques/ 8521 ; DOI : https://doi.org/10.4000/pratiques.8521

Ce document a été généré automatiquement le 15 octobre 2020

(c) Tous droits réservés 


\section{Linguistiques textuelles et discursives}

Introduction: textual and discursive linguistics

Guy Achard-Bayle

1 Nous introduisons ici la partie Linguistique du sommaire consacrée aux textes et aux discours (T\&D). Le but de cette partie est non seulement, comme le dit pour sa part André Petitjean (Voir la contribution d'André Petitjean «Du Centre d'Analyse Syntaxique (CAS) à Praxitexte en passant par le Centre d'Études Linguistiques des Textes et des Discours (Celted) : petite histoire des Sciences du Langage à Metz »), de rappeler et analyser le rôle qu'ont joué ces notions de T\&D à Metz, et les modèles qui vont avec, mais aussi d'interroger la continuité de ces modèles - en particulier aujourd'hui : d'une certaine manière, nous poursuivons de la sorte la réflexion menée lors du colloque de Metz de septembre 2015, sur la «confrontation» de ces deux notions, des domaines qui les illustrent ${ }^{1} \ldots$

2 Nous commençons par donner à cette introduction un tour historique.

3 Que les deux domaines T\&D soient en continuité, de nombreux travaux l'attestent; à commencer par un ouvrage synthétique comme celui de M.-A. Paveau et G.-É. Sarfati (2003) : ainsi ces auteurs réunissent-ils dans leur panorama des tendances ou écoles contemporaines, linguistique textuelle et analyse de discours sous l'appellation « linguistiques discursives » (ibid.).

4 Nous avons pour notre part analysé le rôle qu'a joué J.-M. Adam dans la distinction mais aussi la réunion des deux champs (Achard-Bayle 2014). Le parcours de ce chercheur est à nos yeux « exemplaire " à deux titres : par sa durée et sa ténacité; il en est résulté une certaine complexité, qui a pu être mal appréciée ; nous ne reprendrons pas notre démonstration, préférant renvoyer à J.-M.Adam lui-même et à ce qu'on peut considérer comme une forme d'autoévaluation (Adam 2014a).

5 Toutefois, si l'on veut maintenir la distinction des deux notions, champs ou domaines, ne serait-ce que pour la clarté pédagogique qu'elle permet d'établir dans nos formations ou les formations spécialisées en linguistiques textuelle et discursive, disons 
que la ligne qui les démarquera - plus ou moins nettement - est la part du ou des « contextes » (encyclopédique, situationnel, énonciatif, mémoriel...) $)^{2}$ que l'on prendra ou non en compte dans l'analyse et pour l'interprétation des productions langagières, suivant de ce fait leur étendue. Ainsi, pour rester prudents à un premier niveau d'analyse et d'interprétation mais aussi pour être "pédagogiquement " efficaces comme l'est M. Charolles dès 1978 , complété par $1989^{3}$, on pourra, du côté de la linguistique textuelle, favoriser le niveau qui se situe immédiatement «au-delà de la phrase", pour paraphraser Benveniste (1968). Il s'agit là d'un niveau " macrosyntaxique » ou transphrastique, où se réalisent les opérations qui permettent d'assurer ce que M. Charolles, plus tôt encore $(1976)^{4}$, appelle la « cohérence textuelle proprement dite ».

6 Après quoi, nous pourrions laisser entendre que le domaine du discours concerne - ou ne concerne - que l'analyse et l'interprétation des productions langagières rapportées à leur dimension contextuelle ou, plus strictement encore, à leur dimension sociale... Ce serait une erreur, après ce qu'on vu ou dit des travaux et du parcours de J.-M. Adam, après ce qu'on a dit aussi de la synthèse de M.-A. Paveau et G.-É. Sarfati ; au vu donc de la situation contemporaine où les domaines T\&D sont coordonnés sinon conjoints au sein d'une linguistique « combinée » T\& $D^{5}$...

7 C'est cette situation que feront voir les deux articles qui suivent, dans et par leur enchaînement.

8 Jusque-là, tout en restant au domaine circonscrit plus haut, celui du cotexte, nous nous attacherons à montrer le rôle qu'ont joué dans cette conjonction T\&D, deux chercheurs qui, parmi d'autres, nous sont chers et familiers à Metz, J.-M. Adam et R. Martin ; et, pour représenter et mettre en valeur le domaine circonscrit plus haut, celui du cotexte, nous les commencerons par rappeler leur dette à l'égard de la linguistique tchèque ${ }^{6}$.

9 Cependant nous devons justifier davantage notre choix de ces deux auteurs français. Nous avons écarté celui qui a été sans doute le plus «tchèque » des linguistes de ce que nous avons pu appeler ailleurs « l'École de Prague passant par la Lorraine » (AchardBayle 2013) : B. Combette (1983) dont les ouvrages ouvrages sont publiés chez DuculotDe Boeck et co-édité par Pratiques. Pour notre présente présentation, ce dernier nous a paru précisément «trop " pragois, et nous préférions nous interroger sur ceux, tels R. Martin et J.-M. Adam, dont l'affiliation à la linguistique tchèque ou «pragoise » est plus complexe.

10 Le choix de ces deux auteurs justifié, nous annonçons que nous réduirons encore notre propos en nous focalisant sur R.Martin, car des deux, il est réputé le moins « textualiste ». Cependant cela reste à voir...

11 Mais auparavant, nous effectuons un rappel rapide sur J.-M. Adam. 


\section{Jean-Michel Adam et l'arrière-plan discursif du texte}

12 Si l'on prend pour point de repère l'équation de J.-M. Adam (1989) texte = discours contexte; autrement dit, si nous considérons avec lui que le texte est un discours décontextualisé, (mais rappelons-le, décontextualisé pour les besoins de la cause), il faut bien en déduire, pour la pratique du linguiste, que le discours est à l'arrière-plan de la linguistique textuelle conçue comme méthode d'analyse des textes. J.-M. Adam et d'autres, dont nous-même, avons largement et récemment refait et analysé cet itinéraire? ${ }^{7}$.

13 Pour le reste, les influences du premier Adam, notamment sur les types et séquences de textes, les opérateurs de cohésion textuelle, comme les progressions thématiques, les anaphores, les chaînes de référence..., ne sont pas que pragoises ou néo-pragoises, si l'on songe au fonctionnalisme de M.A. K. Halliday ${ }^{8}$ : M. Bakhtine, R. Barthes, É. Benveniste...

14 Ainsi se clôt notre rapide rappel sur J.-M. Adam. Mais avant de passer à R. Martin, nous redirons un mot du fonctionnalisme; c'est par ce biais que les linguistes de Prague, ou de Brno, mais encore de Bratislava grâce notamment à V. Skalička (1948), ont introduit dans la langue, avec la parole, ce qui deviendra l'élément naturel ${ }^{9}$ de la sémantique logique de R. Martin : « la dynamique communicative » (Martin 1992 [1983], p. 206).

\section{L'arrière-plan « T\&D » de la logique naturelle de Robert Martin}

15 Nous avons déjà traité chez R. Martin (2011) ce que nous avons appelé de la logique du thème; nous nous focaliserons ici sur la question de la distinction et de la complémentarité T\&D.

16 L'ouvrage de logique naturelle de R. Martin (1992 [1983]) parcourt une trajectoire (reprise dans la seconde édition 1992) qui va de la sémantique à la pragmatique ; son projet et même sa revendication (voir le titre de son ouvrage qui débute par : Pour...) est de rassembler in fine deux domaines qui semblaient jusque-là incompatibles: sémantique et pragmatique :

Impossible de traiter en un même lieu des réalités aussi dissemblables [...] Les relations sémantiques sont des relations prévisibles, c'est-à-dire calculables ; il n'en est pas ainsi des relations pragmatiques, dépendantes des situations discursives, aussi variables que les situations elles-mêmes. Comprise comme le lieu du «sens situationnel », la pragmatique peut difficilement être «intégrée ». Elle s'oppose à la sémantique ; elle n'en est pas une partie. (Martin 1992 [1983], p. 15) ${ }^{10}$

17 Entre une sémantique centrée sur la phrase ("lieu des conditions de vérité ») et une pragmatique centrée sur l'énoncé ( « lieu d'actualisation du vrai ou du faux »), l'énoncé comme effectuation et produit de l'énonciation, en interaction et en contexte, autrement dit l'énoncé comme produit discursif; entre elles et eux, donc, s'insère un palier et par là, un lien : le texte.

18 À noter, cependant, que le discours n'est pas cantonné à l'ultime étape du parcours qui va de la sémantique à la pragmatique : il se trouve deux fois, dans les deux dernières 
étapes. En fait, les étapes du parcours et le passage de sémantique à la pragmatique déterminent ce que Martin appelle trois composantes:

- La composante [ou fonction] phrastique, où se déterminent l'acceptabilité formelle (autrement dit grammaticalité) et l'interprétabilité sémantique - autrement dit sémanticité - des unités de communication que constituent les phrases.

- La composante (ou fonction) discursive, où la phrase s'insère dans la cohésion du texte : ainsi, "la cohésion détermine l'appropriation d'une phrase bien formée à un contexte. Un texte répond aux exigences de cohésion si toutes les phrases qu'il comporte y sont acceptées comme des suites possibles du contexte antécédent. »

- La composante pragmatique, où la phrase devenue énoncé, s'interprète dans la situation énonciative. (Martin, 1992 [1983], p. 16, 204-205)

Nous ferons trois remarques à ce propos :

1. Le discours apparaît dès le niveau intermédiaire: en relation avec les opérations - donc l'effet - de cohésion ${ }^{11}$. Ainsi, le discours ne se distingue pas vraiment ou pas encore du texte, du moins à ce niveau ou ce stade. D'ailleurs, à ce stade, R. Martin parle de contexte, là où nous parlerions aujourd'hui de cotexte - mot qui n'apparait pas chez lui.

2.R. Martin distingue bien, par ailleurs, contexte textuel ou transphrastique et contexte situationnel et encyclopédique, et par là la cohésion de la cohérence.

3. Ainsi, il faut comprendre de la manière suivante les trois étapes du parcours des unités de communication: ce que R. Martin appelle (ibid., p. 204) la «phrase hors contexte» est la phrase hors du texte ; la «phrase en contexte » est la phrase comprise (remise) en cotexte; et l'« énoncé », la phrase telle qu'elle est effectuée (actualisée, finalisée) en deux temps, en cotexte et en contexte :

La cohésion textuelle ${ }^{12}$, propre à la composante discursive, se complète [nous soulignons], dans la composante pragmatique, par les exigences de la cohérence ${ }^{13}$. Celle-ci fait intervenir le contexte dans un sens plus large, c'est-à-dire la situation extra-linguistique et les connaissances d'univers. (ibid., p. 205-206)

\section{Pour poursuivre sur l'arrière-plan pragois de Robert Martin...}

19 On voit par ces citations que le propos, les objets d'analyse, et le cadre théorique qui en permettent le traitement, sont ou restent chez R. Martin logico-sémantiques : voir ce qu'André Petitjean a dit du Centre d'Analyse Syntaxique de J. David, R. Martin et G. Kleiber...

Pour autant, ce qui semble inédit dans ce modèle qui distingue bien deux pôles, et se distingue aussi bien à ces deux pôles, c'est qu'il permet en son «milieu ", à la « mitemps » du parcours, de rassembler sémantique et pragmatique, de faire se rejoindre T\&D dans ce que nous appellerons, pour faire écho au sous-titre précédent et à la minisection que nous avons consacrée à J.-M. Adam, l'arrière-plan discursif du texte :

Lieu de la «dynamique communicative» (École de Prague), de la «fonction textuelle " (M.A.K. Halliday), la composante discursive calcule donc l'adéquation de la phrase à son contexte.

En ce sens elle appartient à la sémantique. La cohésion textuelle se fonde sur des critères comme ceux d'isotopie, d'anaphore, de communauté présupositionnelle, dont la fonction s'exerce à l'intérieur même du texte, indépendamment de toute variation situationnelle.

Rien de tel pour la composante pragmatique dont le propre justement est de compléter le calcul sémantique de données qui ne sont pas exclusivement 
linguistiques. Interprétative, elle vise à tenir compte de tout ce qui, dans la situation énonciative, contribue à élaborer la signification: codes autres que linguistiques (gestes, mimiques...); connaissances situationnelles et, plus largement, connaissances d'univers; intentions que le discours recèle ; implicite... (Martin, 1992 [1983], p. 206)

\section{Pour conclure...}

Nous commencerons par tirer de notre étude deux choses :

- Il y a chez Martin (1992 [1983]) deux phases et modes de traitement sémantiques: phrastique et (puis) transphrastique. Le discursif est (appartient au) sémantique.

- La phase 2 ne permet pas de véritable distinction entre T\&D.

Or, dans un premier temps, c'est là que les choses se compliquent, car à la fin du chapitre $\mathrm{V}$ commence à s'estomper, l'opposition entre le domaine des «relations sémantiques", celui du sens prévisible, calculable, et (vs) celui, pragmatique, des interprétations, par principe « aléatoires »:

La pragmatique de l'énoncé est à la fois :

- le lieu où l'énoncé impose un système de représentations, tout un ensemble de croyances, de convictions, une idéologie si l'on veut ;

- le lieu où les représentations, les conceptions de l'univers imposent à l'énoncé une interprétation. (ibid., p. 232)

La distinction ou l'opposition s'estompe donc car il y a dans cette citation une forme de compensation du premier lieu (celui des croyances) par le second (celui des conceptions d'univers).

Mais dans un second temps, R. Martin va plus loin, car on trouve encore cette citation, qui nous a laissé perplexes lors d'un séminaire avec les doctorants sur l'interprétation et nous a paru d'autant plus difficile à démêler qu'elle ne semble pas avoir trouvé de suite dans les recherches de R. Martin (2011) :

Faisons un pas de plus dans ce jardin piégé de la pragmatique et allons jusqu'à la réinterprétation ${ }^{14}$. Cette fois la part de l'imprévisible devient énorme et l'on peut raisonnablement se demander si la pragmatique est autre chose que le terrain vague de l'illusoire. Pourquoi j'ai mal à la tête signifierait-il « Je m'ennuie », plutôt que "Ce problème est difficile " ou bien "J'ai bu un coup de trop »? Tout cela paraît totalement aléatoire et échapper du fait même à toute description scientifique. La réponse cependant n'est pas entièrement négative.

En fait, la pragmatique de la ré-interprétation est au moins partiellement envisageable comme une linguistique du texte. Le texte explicite au moins en partie les données situationnelles. Et c'est dans le texte que deviennent objectivement repérables les éléments indispensables à la ré-interprétation. Il s'y ajoute que certains types de communication sont fortement stéréotypés et, du fait même, le décodage interprétatif peut s'en trouver facilité. (ibid., p. 237)

Malgré la prudence, la modalisation de ses assertions (« au moins partiellement », « au moins en partie ", " peut »...), la question reste donc de savoir si R. Martin envisage (in extremis?) une issue à l'antagonisme et à l'incompatibilité sémantique-pragmatique ; deux remarques encore à ce propos :

- Dans cette dernière citation, le texte apparaît - enfin - à part entière, dans sa stricte acception contemporaine: c'est-à-dire comme le lieu des repérages - lieu et le mode de traitement linguistique objectif des opérations et des effets (qui relèvent ici de la pragmatique). 
- Martin introduit la linguistique des types et/ou des genres discursifs et/ou textuels sous l'expression types de communication.

Pour finir, on peut dire que ces derniers points du parcours et du modèle de R. Martin sont annonciateurs des travaux de la linguistique textuelle «à la française », comme on les retrouvera dans la même décennie, chez Petitjean (1988) puis chez J.-M. Adam (2017 [1992]), ou encore chez les deux ensemble, J.-M. Adam \& A. Petitjean (2005 [1989]).

\section{BIBLIOGRAPHIE}

ABLALI D. et al. (2018). « (Re-)Penser le texte et le discours dans le paysage actuel des sciences du langage ». In Ablali D. et al. (éds). Texte et discours en confrontation dans l'espace européen. Berne : P. Lang, p. 9-32. En ligne : https://hal.univ-lorraine.fr/hal-01872096.

ACHARD-BAYLE, G (dir.). (2006). « Textes et Contextes ». Pratiques 129-130. En ligne : https:// www.persee.fr/doc/prati_0338-2389_2006_num_129_1_2094.

ACHARD-BAYLE, G. (2014). « Texte et discours se comprennent-ils ? ». In : Monte, M. \& Philippe, G. (éds). Genres et textes. Détermination, évolutions, confrontations. Hommage à Jean-Michel Adam. Lyon : Presses universitaires de Lyon, p. 23-38.

ACHARD-BAYLE, G. (2013). « Perspective fonctionnelle de la phrase et linguistique du texte française. L’École de Prague... passant par la Lorraine ». Écho des Études Romanes, VIII (1), p. 65-78. En ligne : https://www.eer.cz/files/2012-1/2012-1-05-Achard.pdf.

ADAM, J.-M. (1989). « Pour une pragmatique linguistique et textuelle ». In : Reichler, C. (éd.). L'Interprétation des textes. Paris : Les Éditions de Minuit, p. 183-222.

ADAM, J.-M. (2006). « Textes/Discours et Co(n)textes ». Pratiques 129-130, p. 21-34. En ligne : https://www.persee.fr/doc/prati_0338-2389_2006_num_129_1_2094

ADAM, J.-M. (2010). « L'émergence de la linguistique textuelle en France (1975-2010) ». Verbum XXXII (2), p. 237-262.

ADAM, J.-M. (2014). « Le paradigme du texte : regard rétrospectif et perspectives pour les sciences des textes ». In : Monte, M. \& Philippe. G. (éds). Genres et textes. Détermination, évolutions, confrontations. Hommage à Jean-Michel Adam. Lyon : Presses universitaires de Lyon, p. 297-323.

ADAM, J.-M. (2015) [1999]. La Linguistique textuelle. Introduction à l'analyse textuelle des discours. Paris : A. Colin.

ADAM, J.-M. (2017) [1992]. Les Texte : types et prototypes. Malakoff : A. Colin.

ADAM, J.-M., \& PETITJEAN, A. (2005) [1989]. Le Texte descriptif. Poétique historique et linguistique textuelle. Paris : A. Colin.

CHARolles, M. (1976). « Grammaire de texte - Théorie du discours - Narrativité ». Pratiques 11-12, p. 133-154. En ligne : https://www.persee.fr/doc/prati_0338-2389_1976_num_11_1_969. 
CHAROLLES, M. (1978). «Introduction aux problèmes de la cohérence des textes. Approche théorique et étude des pratiques pédagogiques ». Langue française 38, p. 7-41. En ligne : https:// www.persee.fr/doc/lfr_0023-8368_1978_num_38_1_6117.

COMBETtEs, B. (1983). Pour une grammaire textuelle. La progression thématique. Bruxelles/Paris : De Boeck/Duculot.

Cusimano, C. (dir.) (2011). « Actualisation - virtualisation ». Études Romanes de Brno 32 (2). En ligne : https://digilib.phil.muni.cz/handle/11222.digilib/114769.

DANEŠ, F. (1974a). « Functional sentence perspective and the organization of the text ». In : Daneš, F. (éd.). Papers on Functional Sentence Perspective. Prague/The Hague : Academia/Éd. Mouton, p. 106-128.

DANEŠ, F. (éd.) (1974b). Papers on Functional Sentence Perspective. Prague/The Hague : Academia/ Éd. Mouton.

FIRBAS, J. (1974). « Some aspects of the Czechoslovak approach to problems of functional sentence perspective ». In : Daneš, F. (éd.). Papers on Functional Sentence Perspective. Prague/The Hague : Academia/Éd. Mouton, p. 10-37.

HALLIDAY, M. A. K., \& HASAN, R. (1976). Cohesion in English. London/New-York/Paris : Longman. MARTIN, R. (1992, [1983]. Pour une logique du sens. Paris : Presses universitaires de France.

MARTIN, R. (2011). « De quelques convictions ». In : Duval, F. (éd.). La « logique » du sens. Autour des propositions de Robert Martin. Metz : Université Paul Verlaine, p. 7-15.

Ministère de l'Éducation nationale, de la Jeunesse et des Sports (2020). PISA (Programme international pour le suivi des acquis des élèves). Mis à jour de févr. 2020. En ligne : https:// www.education.gouv.fr/pid37635/pisa-programme-international-pour-le-suivi-des-acquis-deseleves.html.

Organisation de coopération et de développement économiques (2019). Programme international pour le suivi des acquis des élèves (PISA). Résultat du PISA 2018. En ligne : https://www.oecd.org/pisa/ publications/PISA2018_CN_FRA_FRE.pdf.

PAVEAU, M.-A., \& SARFATI, G.-É. (2003). Les Grandes théories de la linguistique. De la grammaire comparée à la pragmatique. Paris : Armand Colin.

PEŠEK, O. (2010). « La linguistique textuelle tchèque au seuil du XXI ${ }^{e}$ siècle : la genèse d'une discipline et la tradition pragoise ». Verbum XXXII (2), p. 263-282.

PETITJEAN, A. (1988). « Les typologies textuelles ». Pratiques 62, p. 86-125. En ligne : https:// www.persee.fr/doc/prati_0338-2389_1989_num_62_1_1510.

SKALIČKA, V. (1948). « The Need of a Linguistics of la parole ». Recueil linguistique de Bratislava 1, p. 21-38.

SLAKTA, D. (1975). «L'ordre du texte ». Études de Linguistique Appliquée (ÉLA) 19, p. 30-41.

VACHEK, J., (1959). Dictionnaire de linguistique de l'Ecole de Prague. Utrecht/Anvers : Spectrum Éditeurs.

\section{NOTES}

1. Voir Ablali et al. (2018). 


\section{Achard-Bayle (2006).}

3. Ces articles sont des textes fondateurs de la linguistique textuelle en France, sinon "à la française ", et qui portent sur quatre "macro-règles de la cohérence ", restent non seulement d'une parfaite pertinence, mais aussi de la plus grande actualité si l'on considère le rapport annuel du PISA (Programme international pour le suivi des acquis [des élèves]) de l'Organisation de coopération et de développement économiques (OCDE), et particulièrement celui de 2019: d'une part, un critère majeur d'évaluation est la compétence textuelle; d'autre part, les résultats linguistiques obtenus par les élèves de diverses langues-cultures sont fonction de leur maitrise des procédés et des outils qui permettent d'établir (en production) ou restituer (en compréhension) la continuité et l'évolution transphrastique ou encore thématique. Voir les rapports en ligne sur le site de l'Organisation de coopération et de développement économiques (2019) ou celui du Ministère de l'Éducation nationale, de la Jeunesse et des Sports (2020).

4. Texte appelé à faire date comme le $n^{\circ} 11-12$ de la revue, Pratiques, qui le porte! L'intégralité de ce numéro « historique » est en ligne : voir Charolles (1976).

5. Voir l'introduction à Ablali et al. (2018).

6. On pourrait parler, comme J. Vachek (1959) d'École de Prague, mais ce serait oublier, à côté du célèbre Cercle Linguistique de Prague (CLP), le foyer de Brno : autrement dit l'apport de J. Firbas à côté de celui de F. Daneš - même si les deux ont collaboré, notamment en 1974 (à ce propos, voir les travaux récents de Pešek, 2010 et C. Cusimano, 2011).

7. Voir Adam (2014; 2015 [1999]) et Achard-Bayle (2014), mais avant encore Adam (2006).

8. "Néo-pragois » au principe de ses travaux, M. A. K. Halliday revendique, dans les années 1960-1970, l'héritage du CLP (voir notamment Halliday \& Hasan, 1976 ainsi que infra la citation de Martin, 1992 [1983]).

9. Par opposition aux sémantiques logiques formelles. R. Martin (1992 [1983], p. 13), dans son introduction, parle de «l'indigence d'une logique strictement limitée à l'opposition du vrai et du faux ».

10. À quoi pouvait encore faire écho J.-M. Adam $(1989$, p. 187) : «Le "discours" est-il un objet linguistique?»

11. D'où l'importance, y compris en nombre de pages, des sections du chapitre v qu'il consacre au thème, « principal facteur de cohésion » du texte.

12. Note de Martin (1992 [1983]) : « Voir en partic. Charolles 1978 »

13. Autre note de Martin (1992 [1983]) : «Proposition comparable dans Slakta 1975. ». Sur le rôle pionnier de D. Slakta, voir aussi J.M. Adam (2010).

14. Exemple d'interprétation de R. Martin (1992 [1983], p. 232) : Ils se sont mariés bien qu'ils n'aient pas d'enfants «tend à imposer l'idée [présupposition] qu'on ne se marie pas si on n'a pas d'enfants ». 


\section{RÉSUMÉS}

Dans ce texte, qui introduit la partie linguistique du sommaire, nous présentons les contributions de deux linguistes (J.M. Adam et R. Martin) qui ont marqué les sciences du langage telles qu'elles se sont développées et illustrées à Metz à travers la linguistique textuelle ainsi que la sémantique logique et référentielle. Après un rappel de la distinction texte-discours que fait J.-M. Adam au travers de ses multiples travaux, et de leur évolution, nous nous concentrons sur la manière dont cette distinction apparaît, ou non, dans Pour une logique du sens de R. Martin (1992 [1983]). Comme le titre le laisse entendre, l'ouvrage de ce dernier n'est pas au départ un ouvrage de linguistique textuelle et/ou discursive. Néanmoins il le devient à son terme, où le linguiste académicien, après avoir rappelé sa dette envers l'École de Prague, explique son parcours « de la sémantique à la pragmatique ». La démonstration est riche et complexe, et par avance si l'on considère que l'ouvrage paraît à l'orée des années 1980, « hors des sentiers battus » : on verra en particulier que le discours appartient bien au champ sémantique et, inversement, que le texte apparaît à la toute fin de la démonstration, comme une issue à l'aporie de l'(impossible) interprétation (ou « réinterprétation ») pragmatique des énoncés.

In this text, which introduces the linguistic part of the contents, we present the contributions of two linguists (J.M. Adam and R. Martin) who have marked the language sciences as they were developed and illustrated in Metz through textual linguistics as well as logical and referential semantics. After remembering the distinction between text and discourse that J.-M. Adam makes through his multiple works and their evolution, we focus on how this distinction appears, or not, in Pour une logique du sens by R. Martin (1992 [1983]). As the title suggests, this book is not initially a work of textual and/or discursive linguistics. Nevertheless, it becomes so at its end, where the linguist, after recalling his debt to the Prague School, explains how he takes the steps "from semantics to pragmatics". The demonstration is rich, complex, and in advance if we consider that the work appears at the beginning of the 1980s, " out of the box »: we will see in particular that the discourse belongs to the semantic field and, conversely, that the text appears at the very end of the demonstration, as an outcome from the aporia of the (impossible) pragmatic interpretation (or "re-interpretation") of the statements.

\section{INDEX}

Mots-clés : linguistique textuelle, linguistique discursive, sémantique logique, pragmatique de l'interprétation

Keywords : textual linguistics, discursive linguistics, logical semantics, pragmatics of interpretation

\section{AUTEUR}

\section{GUY ACHARD-BAYLE}

Université de Lorraine, Crem, F-57000 Metz, France 